\section{Versão brasileira do Instrumento de Avaliação da Dor em Paciente Não Comunicativo (NOPPAIN): equivalência conceitual, de itens e semântica}

\author{
Brazilian version of the Non-communicative \\ Patient's Pain Assessment Instrument (NOPPAIN): \\ conceptual, item, and semantic equivalence
}

\section{Abstract}

The evaluation and measurement of pain in individuals with dementia and unable to communicate verbally has been a challenging experience. The Non-communicative Patient's Pain Assessment Instrument (NOPPAIN) is an instrument that evaluates this phenomenon by observing behaviors that express pain. Considering the lack of instruments for this purpose in Brazil, the current study was designed to translate the NOPPAIN instrument into Brazilian Portuguese and culturally adapt it to the Brazilian reality. This was a methodological study, with Guillemin et al. as the theoretical reference. The study included four steps: translation; obtaining a consensus version; back-translation; and evaluation of the translation and back-translation by an expert panel. In this initial approach, the NOPPAIN-Br showed semantic equivalence to the original instrument and is now available in Brazilian Portuguese for further validation.

Pain Measurement; Dementia; Aged
Raquel Soares De Araujo 1

Lilian Varanda Pereira 1

\section{Introdução}

A mensuração da experiência dolorosa é tarefa desafiadora para aqueles que procuram manejála adequadamente, quer pela complexidade do fenômeno doloroso ou falta de um instrumento de medida ideal, que possibilite acesso preciso e acurado ao que o outro está sentindo 1,2,3.

Nos casos de incapacidade cognitiva grave e impossibilidade de comunicação verbal das sensações, soma-se a impossibilidade de utilizar o autorrelato, padrão-ouro para reconhecer, avaliar a tratar a dor nas populações. Nestes casos, os instrumentos que se valem da observação de comportamentos que expressam dor, como a expressão facial, as verbalizações e vocalizações, os movimentos corporais, as mudanças nas interações interpessoais, nas atividades, rotinas e no estado mental 4,5,6,7, ajudam a reduzir riscos de interpretação errônea de mais ou de menos daquilo que a pessoa está vivenciando 2,3.

Instrumentos para a medida da dor por meio de comportamentos observáveis, que estão no domínio involuntário e não intencional, dependendo mais de formas automáticas de expressão da dor, estão sendo propostos 5,8,9,10,11,12, como o Non-communicative Patient's Pain Assessment Instrument (NOPPAIN), original do inglês, elaborado por Snow et al. 1 .

O NOPPAIN é um instrumento simples e clinicamente útil para mensurar a dor a partir do julgamento de comportamentos que a expres- 
sam, acessível aos profissionais de diferentes áreas e níveis de formação 11,12,13,14,15. Foi traduzido para o italiano por Ferrari et al. ${ }^{13}$ e validado por Novello et al. 14 e Horgas et al. 15. Consiste de quatro seções, que encampam nove figuras de situações de cuidados diários; seis figuras que indicam comportamentos de dor; e a figura de uma escala descritiva de intensidade subjetiva de 11 pontos ( 0 - 10$)$, onde 0 (zero) significa "sem dor" e 10 (dez) "a pior dor" associados a cinco descritores: "nenhuma dor", "dor leve”, "moderada", "forte” e "pior dor". Inicialmente o observador deve indicar os cuidados realizados; após, os comportamentos de dor observados durante os cuidados e a intensidade de cada um deles; e por fim, a intensidade global da dor no pior momento. O somatório dos escores de cada seção indicará o escore NOPPAIN de dor.

Instrumentos de medida elaborados na cultura de origem ou adaptados culturalmente do idioma original para o da população-alvo possibilitam a obtenção de dados referentes à dor em diferentes culturas, viabilizando comparações transculturais sobre a experiência dolorosa, avançando nessa área do conhecimento 16 . Ademais, a avaliação da dor é fundamental para tratamento precoce e eficaz, um dos direitos humanos 17 .

Assim, este estudo foi proposto e teve como objetivo: descrever os resultados da equivalência conceitual, de itens e semântica entre o NOPPAIN original em inglês, e a versão em português brasileiro para avaliação da dor em pacientes não comunicativos.

\section{Metodologia}

Estudo de adaptação transcultural do Instrumento de Avaliação da Dor em Paciente Não Comunicativo (NOPPAIN), para o português brasileiro, que recebeu parecer favorável do Comitê de Ética em Pesquisa da Universidade Federal de Goiás (protocolo $\mathrm{n}^{\mathrm{o}}$. 0122010) e consentimento de seus proponentes.

O referencial utilizado foi o proposto por Guillemin et al. 16 , sendo percorridos quatro dos cinco passos propostos:

- Passo 1: tradução do NOPPAIN para o português brasileiro, língua-mãe das duas bilíngues que trabalharam de forma independente;

- Passo 2: as duas versões no português brasileiro foram analisadas por uma equipe de bilíngues e pesquisadores, surgindo a Versão de Consenso do NOPPAIN para o idioma-alvo-o NOPPAIN-BrVCLP. Os termos, frases e palavras do NOPPAIN foram categorizados em 64 elementos, listados na primeira coluna da Tabela 1;
- Passo 3: foram feitas quatro retraduções do NOPPAIN-Br-VCLP, por quatro bilíngues, com língua-mãe no inglês, de forma independente. Todos eram ingênuos quanto aos objetivos do estudo. Resultaram as versões RT1, RT2, RT3 e RT4;

- Passo 4: o NOPPAIN-Br-VCLP e as quatro retraduções foram analisadas pela comissão de especialistas, composta por seis bilíngues, incluindo profissionais da área de saúde (médicos neurologistas e enfermeiros) e linguistas, conhecedores do assunto em questão (medida de dor, comportamento de dor, idoso, demência e processo de adaptação transcultural de instrumentos para o português brasileiro). Para análise da concordância sobre a adequação dos elementos do NOPPAIN-Br-VCLP utilizou-se uma escala de 0 (zero) a 10 (dez), onde o escore 0 (zero) significava nenhuma concordância; escores 1 (um), 2 (dois), 3 (três) e 4 (quatro), pouca concordância; escores 5 (cinco) e 6 (seis), média concordância; escores 7 (sete), 8 (oito) e 9 (nove), concordância boa; e escore 10 (dez), concordância muito boa. O resultado foi explorado pela média aritmética dos escores atribuídos a cada elemento. Na análise das retraduções a porcentagem de concordância entre os especialistas foi o direcionador da escolha da melhor versão. Desse processo resultou a Versão de Consenso Retraduzida (NOPPAINBr-VCRT) e a Versão Pré-Final do NOPPAIN para o idioma-alvo (NOPPAIN-Br-VPF), denominada NOPPAIN-Br.

\section{Resultados}

Os elementos do NOPPAIN-Br-VCLP foram descritos na segunda coluna da Tabela 1 . Houve correções, como "Did you see pain?", traduzido como "Você viu dor?", substituído por "Você notou dor?" e finalmente por "Você observou dor?".

O termo "sponge bath", traduzido como "banho de esponja", que significa dar banho no indivíduo em sua própria cama, foi traduzido como "banho de leito", expressão utilizada na cultura brasileira.

A instrução "Rate the resident's pain at the highest level you saw it today" foi traduzida como "Avalie a dor do paciente, no nível mais alto que você a notou hoje" e substituída por “....você a observou durante a realização dos cuidados", pois os comportamentos de dor serão julgados após a realização desses cuidados.

O resultado da comparação dos elementos do NOPPAIN-Br-VCLP com o NOPPAIN original, e desse com as retraduções, resultaram na Versão Retraduzida de Consenso (NOPPAIN-BrVCRT) e Versão Pré-Final no Português Brasi- 
Elementos do Non-communicative Patient's Pain Assessment Instrument (NOPPAIN), original em inglês, tradução para o português brasileiro e retradução.

\section{Versão original em inglês (NOPPAIN)}

Versão de consenso em português brasileiro (NOPPAIN-Br-VCLP)

\section{Non Communicative Patient's Pain Assessment Instrument \\ Directions: Nursing staff member should complete at least 5 minutes of daily care activities for the resident while observing for pain behaviors. This form should be completed immediately following care activities}

Activity chart check list

Did you do this?

Did you see pain when you did this?

Check "yes" or "no" for each item in columns A and B

(a) Put resident in bed OR saw resident lying down

(b) Turned resident in bed

(c) Transferred resident (bed to chair, chair to bed, standing or wheelchair to toilet)

(d) Sat the resident up (bed or chair) OR saw resident sitting

(e )Helped resident stand OR saw resident stand

(f) Dressed resident

(g) Fed resident

(h) Helped resident walk OR saw resident walk

(i) Bathed resident OR gave resident sponge bath

SCORING:

Add up the number of "yes" boxes you checked in column B

Pain behavior

What did you see or hear during care?

Pain words?

That hurts!

Ouch!

Stop that!

Did you see this?

How intense were the pain words?

Lowest possible intensity

Highest possible intensity

Pain faces?

Grimaces

Winces

Furrowed brow
Instrumento de Avaliação da Dor em Paciente Não Comunicativo

Instruções: O membro da equipe de enfermagem deverá fazer a avaliação, durante pelo menos

5 minutos de atividades dos cuidados diários

prestados ao residente (paciente), enquanto observa por comportamentos de dor. Este formulário deve ser preenchido imediatamente seguindo as atividades de cuidado

Prontuário para check list de atividades Você fez isso?

Você notou dor quando fez isso?

Marque sim ou não para cada item nas colunas A e B

(a) Colocou o residente (paciente) na cama OU viu o residente (paciente) se deitar

(b) Virou o residente (paciente) no leito

(c) Transferiu o residente (paciente) (do leito para cadeira, da cadeira para leito, levantou ou levou-o de cadeira de rodas para o banheiro)

(d) sentou o residente (paciente), (cadeira ou leito) OU viu o residente (paciente) sentando-se

(e) Ajudou o residente (paciente) a ficar de pé OU viu o residente (paciente) ficar de pé

(f) Vestiu o residente (paciente)

(g) Alimentou o residente (paciente)

(h) Ajudou o residente (paciente) caminhar OU viu o residente caminhar

(i) Deu banho de chuveiro no residente (paciente) OU deu banho de leito no residente (paciente)

$$
\text { Pontuando: }
$$

Some o numero de caselas que você marcou na coluna B, que contenham a palavra "sim" Comportamento de dor

O que você viu e ouviu durante o cuidado? Palavras de dor? Isto dói! Aiaiai!

Pare com isso!

Você observou isso?

Quão intensas foram as palavras de dor? Menor intensidade possível

Maior intensidade possível

Fácies de dor? Caretas

Contrações (contorções) Testa franzida
Versão retraduzida de consenso (NOPPAIN-VCRT)

Non-Communicative Patient's Pain Assessment Instrument

Directions: Nursing staff member should complete at least 5 minutes of daily care activities for the resident (patient) while observing for pain behaviors. This form should be completed immediately following care activities

\section{Activity form check list} Did you do this?

Did you notice pain when you did this? Check "yes" or "no" for each item in columns A and $B$

A. Put patient in bed OR saw patient lying down

$$
B \text {.Turned the patient in bed }
$$

C. Transferred the patient from the bed to the chair, from the chair to the bed, picked the patient up, or took the patient in a wheelchair to the bathroom $D$. Sat up the patient, (chair or bed) OR saw the patient sitting down

E. Helped patient stand or saw patient stand

$$
\text { F. Dressed patient }
$$$$
\text { G. Fed patient }
$$

$H$. Helped patient walk or saw patient walk

I. Showered patient or gave patient sponge bath

\section{SCORING:}

Add up the number of "yes" boxes you checked in column B

Pain behavior

What did you see or hear during care?

$$
\begin{gathered}
\text { Pain words? } \\
\text { That hurts! } \\
\text { Ouch! }
\end{gathered}
$$

Stop that!

Did you see this?

How intense were the pain words?

Lowest possible intensity

Highest possible intensity

Pain faces?

Grimaces

Winces

Furrowed brow

(continua) 
Tabela 1 (continuação)

Versão original em inglês (NOPPAIN) $\quad$ Versão de consenso em português brasileiro
(NOPPAIN-Br-VCLP)

How intense were the pain faces?

Pain noises?

Moans

Groans

Grunts

Cries

Gasps

Sighs

How intense were the pain noises?

Bracing?

Rigidity

Holding

Guarding

How intense was the bracing?

Rubbing?

Massaging affected area?

How intense was the rubbing?

Restlessness?

Frequent shifting

Rocking

Inability to stay still

How intense was the restlessness?

Add up the number of "yes" boxes you checked

Add up the numbers you circled on the intensity scales

Pain intensity

Rate the resident's pain at the highest level you saw it today

No pain

Mild

Moderate

Severe

Worst pain possible

If a resident reported they have pain, or their NOPPAIN score is 3 or more, report the resident to the nurse for a comprehensive exam

NOPPAIN scoring

Total
Quão intensa foi a face de dor?

Ruídos de dor?

Gemidos

Murmúrios

Grunhidos

Choro

Ofegar (respiração ofegante)

Suspiros

Quão intensos foram os ruídos de dor?

Segurando? Apoiando? Suportando? OBS: posição

antálgica (posição para aliviar dor)

Rigidez

Segurando

Protegendo

Quão intensos foram os movimentos antálgicos? Friccionando?

Massageando área afetada

Quão intensa foi a fricção? Inquietação?

Posicionamento frequente

$$
\text { Balançando }
$$

Inabilidade de se manter parado

Quão intensa foi a inquietação?

Some o numero de caselas que você marcou que

$$
\text { contenham a palavra sim }
$$

Some os números que você circulou nas escalas de intensidade

Intensidade da dor

Avalie a dor do paciente, no nível mais alto que você a notou durante o cuidado

Nenhuma dor

$$
\text { Leve }
$$

Moderada

Forte

Pior dor possível

Se o paciente relatou dor, ou a pontuação no NOPPAIN dele for igual ou maior que 3 , refira o paciente ao enfermeiro para um exame de maior abrangência

Pontuando NOPPAIN

Total
How intense were the pain faces?

Pain noises?

Moans

Groans

Grunts

Cries

Gasps

Sighs

How intense were the pain noises? Bracing? Supporting?

$$
\begin{aligned}
& \text { Rigidity } \\
& \text { Holding } \\
& \text { Guarding }
\end{aligned}
$$

How intense was the bracing?

$$
\text { Rubbing? }
$$

Massaging affected area

How intense was the rubbing?

Restlessness?

Frequent shifting

Rocking

Inability to stay still

How intense were the restlessness?

Add up the number of "yes" boxes you checked

Add up the numbers you circled on the intensity scales

Pain intensity

Evaluate patient's pain at the highest level you saw during care activities

$$
\begin{gathered}
\text { No pain } \\
\text { Mild } \\
\text { Moderate } \\
\text { Strong }
\end{gathered}
$$

Worst possible pain

If a resident reported they have pain, or their NOPPAIN score is 3 or more, report the resident to the nurse for a comprehensive exam

\section{NOPPAIN scoring}

Total 
leiro (NOPPAIN-VPF), cujos elementos estão listados nas colunas dois e três da Tabela 1, respectivamente.

Dos 64 elementos de cada retradução, 59,4\% elementos alcançaram $100 \%$ de concordância entre os especialistas; 16 (25\%) alcançaram 83,3\% de concordância, 14,1\% 66,6\%. O elemento "Transferred resident (bed to chair, chair to bed, standing or wheelchair to toilet)" teve concordância de $50 \%$, permanecendo a versão "Transferred the patient from the bed to the chair, from the chair to the bed, picked the patient up, or took the patient in a wheelchair to the bathroom".

Quanto ao NOPPAIN-Br-VCLP, dos 64 elementos, 56,3\% alcançaram média 10 (dez), equivalente à concordância "muito boa" e 43,7\%, média entre 7,0 e 9,9 - concordância "boa".

A tradução do termo "check list", de "Activity chart check list" foi "verificação", alcançando $50 \%$ de concordância entre os juízes.

O NOPPAIN-Br-VPF foi nomeado Instrumento de Avaliação da Dor em Pacientes Não Comunicativos (NOPPAIN-Br) (Figura 1).

\section{Discussão}

A mensuração da dor em pessoas com déficit cognitivo e de comunicação tem sido uma realidade nos últimos 15 anos, em países da Europa, Austrália e Estados Unidos da América 3,5,8,9,10,11,12,13,14, no entanto, ainda não estão disponíveis no idioma português brasileiro, o que faz deste estudo pioneiro na adaptação e disponibilização de um instrumento para medir dor em brasileiros com demência avançada.
No processo de obtenção do NOPPAIN-BrVCLP e NOPPAIN-Br-VCRT observou-se que as traduçõeseretraduçõesforammuitosemelhantes. Algumas palavras foram apenas substituídas por sinônimos, outras corrigidas gramaticalmente.

O processo de equivalência conceitual e de itens do NOPPAIN não exigiu mudanças na ordem dos elementos, retirada ou acréscimo de itens. Trata-se de escala de fácil entendimento, com desenhos simples e frases curtas, que foram traduzidas e retraduzidas mantendo equivalência com a nova versão, fundamental para a introdução de instrumentos em novas culturas 16 .

Novas pesquisas, com amostras representativas, estão sendo conduzidas para complementar esta primeira abordagem da adaptação transcultural do NOPPAIN para o idioma português brasileiro, incluindo os processos de equivalência operacional, de mensuração e funcional do instrumento. Estes estudos serão necessários para que um instrumento de mensuração da dor em pessoas com déficit cognitivo grave e incapacidade de comunicação possa ser disponibilizado na cultura brasileira.

\section{Conclusão}

Durante as etapas percorridas nesta abordagem inicial, o NOPPAIN-Br atingiu os critérios de equivalência conceitual, de itens e semântica, fundamentais para a continuidade do processo de adaptação transcultural desse instrumento. 
Figura 1

Versão brasileira do Instrumento de Avaliação da Dor em Paciente Não Comunicativo (NOPPAIN-Br).

NOPPAIN-Br

(Versão brasileira do Instrumento de Avaliação da Dor em Paciente Não Comunicativo)

\begin{tabular}{|r|r|}
\hline Nome do avaliador: & \\
\hline Nome do paciente: & \\
\hline Data: & \\
\hline Horário: & \\
\hline
\end{tabular}

Instruções: o profissional de enfermagem deve completar pelo menos 5 minutos de cuidados diários para o paciente, enquanto observa os comportamentos de dor. Ambas as páginas deste formulário devem ser completadas imediatamente após as atividades de cuidado.

\begin{tabular}{|c|c|c|c|}
\hline \multicolumn{2}{|c|}{$\begin{array}{l}\text { I. LISTA DE VERIFICAÇÃO DE } \\
\text { ATIVIDADES } \\
\text { Marque "Sim" ou "Não" para cada } \\
\text { item nas colunas A e B }\end{array}$} & $\begin{array}{c}\text { A } \\
\text { Você fez } \\
\text { isso? }\end{array}$ & $\begin{array}{c}\text { B } \\
\text { Você } \\
\text { observou } \\
\text { dor } \\
\text { durante } \\
\text { esta } \\
\text { atividade? }\end{array}$ \\
\hline $\begin{array}{l}\text { A. Colocou o paciente } \\
\text { na cama OU viu o } \\
\text { paciente se deitar }\end{array}$ & 8 & \begin{tabular}{|l|}
$\mathrm{S}$ \\
$\mathrm{N}$
\end{tabular} & \begin{tabular}{|l|}
$\mathrm{S}$ \\
$\mathrm{N}$
\end{tabular} \\
\hline $\begin{array}{l}\text { B. Virou o paciente } \\
\text { no leito }\end{array}$ & & \begin{tabular}{|l|}
$\mathrm{S}$ \\
$\mathrm{N}$ \\
\end{tabular} & \begin{tabular}{|l|}
$\mathrm{S}$ \\
$\mathrm{N}$ \\
\end{tabular} \\
\hline $\begin{array}{l}\text { C. Transferiu o paciente } \\
\text { (do leito para cadeira, } \\
\text { da cadeira para leito, } \\
\text { de pé ou de cadeira de } \\
\text { rodas para o banheiro) }\end{array}$ & & \begin{tabular}{|l|}
$\mathrm{S}$ \\
$\mathrm{N}$ \\
\end{tabular} & \begin{tabular}{|l|}
$\mathrm{S}$ \\
$\mathrm{N}$ \\
\end{tabular} \\
\hline $\begin{array}{l}\text { D. Sentou o paciente } \\
\text { (cadeira ou leito) OU } \\
\text { viu o paciente se } \\
\text { sentando }\end{array}$ & & $\mathrm{N}$ & \begin{tabular}{|l|}
$\mathrm{S}$ \\
$\mathrm{N}$ \\
\end{tabular} \\
\hline $\begin{array}{l}\text { E. Ajudou o paciente } \\
\text { a ficar de pé OU viu o } \\
\text { paciente ficar de pé }\end{array}$ & & \begin{tabular}{|l|}
$\mathrm{S}$ \\
$\mathrm{N}$ \\
\end{tabular} & \begin{tabular}{|l|}
$\mathrm{S}$ \\
$\mathrm{N}$ \\
\end{tabular} \\
\hline F. Vestiu o paciente & 終 & \begin{tabular}{|l|}
$\mathrm{S}$ \\
$\mathrm{N}$ \\
\end{tabular} & \begin{tabular}{|l|}
$\mathrm{S}$ \\
$\mathrm{N}$ \\
\end{tabular} \\
\hline $\begin{array}{l}\text { G. Alimentou o } \\
\text { paciente }\end{array}$ & $\mid$ & \begin{tabular}{|l|}
$\mathrm{S}$ \\
$\mathrm{N}$ \\
\end{tabular} & \begin{tabular}{|l|}
$\mathrm{S}$ \\
$\mathrm{N}$ \\
\end{tabular} \\
\hline $\begin{array}{l}\text { H. Ajudou o paciente } \\
\text { a caminhar OU viu o } \\
\text { paciente caminhar }\end{array}$ & & \begin{tabular}{|l|}
$\mathrm{S}$ \\
$\mathrm{N}$ \\
\end{tabular} & \begin{tabular}{|l|}
$\mathrm{S}$ \\
$\mathrm{N}$ \\
\end{tabular} \\
\hline $\begin{array}{l}\text { I. Deu banho de } \\
\text { chuveiro no paciente } \\
\text { OU deu banho de } \\
\text { leito no paciente }\end{array}$ & 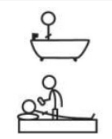 & \begin{tabular}{|l|}
$\mathrm{S}$ \\
$\mathrm{N}$ \\
\end{tabular} & \begin{tabular}{|l|}
$\mathrm{S}$ \\
$\mathrm{N}$ \\
\end{tabular} \\
\hline $\begin{array}{l}\text { Pontuação: } \\
\text { Some o número de case } \\
\text { marcou na coluna B, que } \\
\text { "Sim" (S) }\end{array}$ & $\begin{array}{l}\text { s que você } \\
\text { contenham }\end{array}$ & & \\
\hline
\end{tabular}

Se o paciente relatou dor, ou a pontuação do NOPPAIN-Br for 3 , comunique a condição ao enfermeiro para um exame mais abrangente.

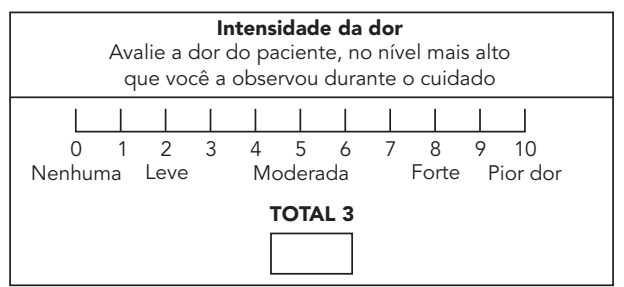

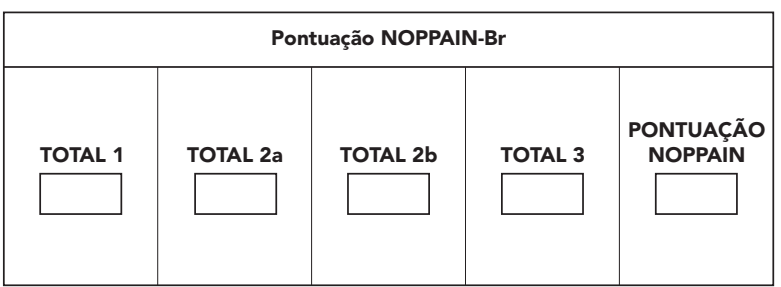




\section{Resumo}

A mensuração da dor em pessoas com déficit cognitivo grave, inábeis em comunicar verbalmente o que estão sentindo, tem sido um desafio. O Instrumento para Avaliação da Dor em Paciente Não Comunicativo (NOPPAIN) é um instrumento que propõe a mensuração da dor por meio do julgamento de comportamentos observados, que expressam tal experiência. A inexistência de instrumentos desse tipo em nossa cultura levou ao desenvolvimento deste estudo, que teve como objetivo: adaptar culturalmente o NOPPAIN para o idioma português brasileiro. Trata-se de estudo metodológico, realizado com base no referencial de Guillemin et al. Foram percorridos quatro passos: tradução para o português brasileiro, obtenção de uma versão de consenso, retradução e avaliação das versões traduzidas e retraduzidas por um comitê de especialistas. Nesta abordagem inicial, o NOPPAIN-Br apresentou equivalência semântica com o original e está disponível no idioma português brasileiro para validação complementar

Medição da Dor; Demência; Idoso

\section{Colaboradores}

R. S. De Araujo e L. V. Pereira participaram do processo de planejamento da investigação, coleta e análise de dados e redação do artigo.

\section{Referências}

1. Snow AL, Weber JB, O'Malley KJ, Cody M, Beck C, Bruera E, et al. NOPPAIN: a nursing assistantadministered pain assessment Instrument for use in dementia. Dement Geriatr Cogn Disord 2004; 17:240-6.

2. Hadjistavropoulos T, Fine PG. Chronic pain in older persons: prevalence, assessment and management. Rev Clin Gerontol 2006; 16:231-41.

3. McAuliffe L, Nay R, O’Donnell M, Fetherstonhaugh D. Pain assessment in older people with dementia: literature review. J Adv Nurs 2009; 65:2-10.

4. Beck CK, Vogelpohl TS. Problematic vocalizations in institutionalized individuals with dementia. J Gerontol Nurs 1999; 25:17-26.

5. Feldt KS. The checklist of nonverbal pain indicators (CNPI). Pain Manag Nurs 2000; 1:13-21.

6. Kovach CR, Noonan PE, Griffi J, Muchka S, Weissman DE. The assessment of discomfort in dementia protocol. Pain 2002; 3:16-27.

7. Weiner DK, Peterson B, Ladd K, McConnell E, Keefe F. Pain in nursing home residents: An exploration of prevalence, staff perspectives, and practical aspects of measurement. Clin J Pain 1999; 15:92-101.
8. Hadjistavropoulos T, Craig KD. A theoretical framework for understanding selfreport and observational measures of pain: a communications model. Behav Res Ther 2002; 40:551-70.

9. Fuchs-Lacelle S, Hadjistavropoulos T. Development and preliminary validation of the Pain Assessment Checklist for Seniors with Limited Ability to Communicate (PACSLAC). Pain Manag Nurs 2004; 5:37-49.

10. Warden V, Hurley AC, Volicer L. Development and psychometric evaluation of the PAINAD (Pain Assessment in Advanced Dementia) scale. J Am Med Dir Assoc 2003; 4:9-15.

11. Herr K, Bjoro K, Decker S. Tools for assessment of pain in nonverbal older adults with dementia: a state-of-the-science review. J Pain Symptom Manage 2006; 31:170-92.

12. Schofield P. Assessment and management of pain in older adults with dementia: a review of current practice and future directions. Curr Opin Support Palliat Care 2008; 2:128-32. 
13. Ferrari R, Martini M, Mondini S, Novello C, Palomba D, Scacco C, et al. Pain assessment in noncommunicative patients: the Italian version of the Non-Communicative Patient's Pain Assessment Instrument (NOPPAIN). Aging Clin Exp Res 2009; 21:298-306.

14. Novello C, Ferrari R, Scacco C, Visentin M. The Italian version of NOPPAIN scale: validation during education. Assist Inferm Ric 2009; 28:198-205.

15. Horgas AL, Nichols AL, Schapson CA, Vietes K. Assessing pain in persons with dementia: relationships among the Non-communicative Patient's Pain Assessment Instrument, self-report, and behavioral observations. Pain Manag Nurs 2007; 8:77-85.
16. Guillemin F, Bombardier C, Beaton D. Cross-cultural adaptation of health-related quality of life measures: literature review and proposed guidelines. J Clin Epidemiol 1993; 46:1417-32.

17. Joint Commission Resources. Facts about pain management. http://www.jointcommission.org/ assets/1/18/Pain_Management.pdf (acessado em 12/Jan/2010).

Recebido em 22/Ago/2011

Versão final reapresentada em 27/Jun/2012 Aprovado em 13/Jul/2012 